\title{
Trapped in the Wrong Theory: Rethinking Trans Oppression and Resistance
}

Resistance hardly ever has a straightforward public presence. It is rather duplicitous, ambiguous, even devious. But it is almost always masked and hidden by structures of meaning that countenance and constitute domination. - María Lugones (2003, x)

s I started my transition during the mid-nineties, I searched, almost desperately, for a story that worked for me. For the longest time, I thought I needed a story that secured my claims to womanhood and that illuminated my (often confusing) life experiences. How else to justify my claims? How else to understand my experiences? At the time, unfortunately, there were really only two stories available for me to draw on. Alas, there are still only two stories to draw on.

In what I call the "wrong-body" model, transsexuality involves a misalignment between gender identity and the sexed body. This idea developed in the context of sexology, medicine, and psychiatry (facilitated by technological developments). ${ }^{1}$ While in the psychological variant of the model, transsexuality is viewed as a problem of the mind (albeit treatable through sex reassignment surgery) by the medical establishment, in the wrong-body model proper, transsexuality is viewed as a problem of the body by transsexuals themselves. The wrong-body model proper has two versions. In the weak version, one is born with the medical condition of transsexuality and then, through genital reconstruction surgery, becomes a woman or a man (in proper alignment with an innate gender identity). In the strong version, one's real sex is determined by gender identity. On the basis of this native identity one affirms that one has always really been the woman or a man that one claims to be. In both versions, one is effectively a man or woman "trapped in the wrong body."

By the mid-1990s a new story had also begun to emerge. While Sandy Stone's (1991) groundbreaking work gave birth to trans studies, Leslie

\footnotetext{
Special thanks to an anonymous reviewer whose trenchant critique of an earlier draft helped transform this into a much stronger essay.

${ }^{1}$ See Meyerowitz $(2002,14-50)$.

${ }^{2}$ See Rubin $(2003,150-51)$.

[Signs: Journal of Women in Culture and Society 2014, vol. 39, no. 2]

(C) 2013 by The University of Chicago. All rights reserved. 0097-9740/2014/3902-0005\$10.00
} 
Feinberg (1993) and Kate Bornstein (1994) laid the foundations for the new transgender politics. For the first time, trans people were theorizing themselves for themselves. This new vision witnessed the introduction of the word "transgender" as a broad umbrella term-bringing together different gender-variant people (including transsexuals, cross-dressers, drag queens and kings, and others). In this way, it was a story that aimed for unity in the face of hostility toward those who were gender nonnormative. The new vision subscribed to a "beyond the binary" model. It claimed that because transgender people don't fit neatly into the two dichotomous categories of man and woman, attempts are made to force them into this binary system. The medical regulation of transsexuality, in this account, is one of the main ways that society tries to erase transgender people. ${ }^{3}$

Back then, already, I felt deeply suspicious of the wrong-body account. For one thing, I disliked its pathologizing aspects. Yet I also worried about the new one. For most of my life I'd felt problematically positioned with respect to the binary. This was a horrible feeling: what made me feel well was being recognized as a woman. Ambivalent about both models, I simply found friendship and love in the activist trans subcultures of Los Angeles. There we had developed alternative ways of living gender that felt far more safe and healthy. And once I had fully immersed myself in these cultures, the alternative accounts didn't matter so much.

Many years have gone by, however, and I've become increasingly motivated to provide an account that truly satisfies me. Having learned from my experiences, I'm interested in a story that's actually informed by my own life as it's been given meaning within local trans subcultures. I desire an account that captures my own reality and the reality of my chosen family, friends, and lovers - a story that is founded upon my experiences in trans community, my local knowledge. In this article, I begin to develop that account. To be clear, I don't think it's the only good one. And I certainly don't claim that it's universally applicable. Yet I do think the story has some use in that it can help get us beyond some tough political impasses. Before I begin the account, then, I'll sketch out some of the key issues.

\section{What's at stake?}

Despite the dominance of the transgender paradigm in both trans studies and trans politics (at least in Anglo-American circles), there have been thinkers who have raised serious worries about this particular vision. Jay Prosser (1998), Henry Rubin (2003), and Viviane K. Namaste (2005) have, all in

${ }^{3}$ This account is admittedly simplified. 
their own ways, worried that it doesn't accurately capture the realities of transsexual people. ${ }^{4}$ One of the worries has been the transgender paradigm's alleged commitment to the view that gender is a mere cultural construction, which runs counter to the actual experiences of transsexual people for whom gender identity seems impervious to cultural modification (Prosser 1998, 7). ${ }^{5}$ Another concern is that the model provides a vision of politics that doesn't leave space for trans people who don't self-identity as beyond the binary. Many trans people see themselves as men and women. Taken to its most extreme, the beyond-the-binary model suggests these people are mistaken (i.e., it invalidates their self-identities). At best, it accepts such self-identifications while recognizing them as politically problematic since they disavow the resistant force of trans lives lived in opposition to the oppressive binary (Namaste 2005, 7).

These concerns have been manifested politically in different ways. First, "transsexual" can now function as a way to distance oneself from and oppose the political vision articulated in the transgender model. For example, various forms of transsexual separatism have recently been voiced in the United States, mostly on online social networking sites and blogs. In this type of political view, transsexuals - particularly those who have undergone genital reconstruction and who choose not to disclose their trans history - see themselves as nonconsensually subsumed under the transgender umbrella and wrongly associated with gender nonnormative people (such as cross-dressers). ${ }^{6}$ Second, while the beyond-the-binary model is invariably cited as the underlying political vision of transgender politics, many self-identified transgender people also self-identify as men and women. Consequently, categories such as "genderqueer" have emerged to capture those who actually live in opposition to the binary, while "trans" " is deployed to include not only those who identify as men and women but also those who do not. The irony is obvious. "Transgender" was originally deployed as an umbrella term for those "beyond the binary," yet the dominance of self-identified transgender men and women who do political work under the category "transgender" has required a new iteration of the beyond-the-binary vision. What this demonstrates, in part, is a serious disconnect between the theory cited and the actual practice.

As it stands, transgender politics now sometimes appeals to both the beyond-the binary model and the wrong-body model. While not originally

${ }^{4}$ See Namaste for an argument that the transgender model is imperialistic in nature (2005, 103-26).

${ }^{5}$ See Salamon (2010) for a strong defense of constructionism against nativism. Julia Serano $(2007,82-83)$ proposes a nativist view.

${ }^{6}$ See Cooke (2007) for an example of this view. 
political, the latter can shed its pathologizing aspects and thereby serve as the basis for a politics that is roughly analogous to a politics of ability: there are some people who have congruence between identity and body (cissexuals) and others who do not or who did not (transsexuals), but the social world is constructed in such a way that it privileges the former. ${ }^{7}$

Notably, however, the wrong-body model fails to secure the validity of trans claims to belong in a particular sex. This is particularly bad for the model, since this is precisely what it purports to secure. Consider the multiplicity of features relevant to sex determination: chromosomes, genitalia, gonads, hormone levels, reproductive capacity, and so forth. In order to say that a transsexual (after genital reconstruction surgery) has undergone a "sex change," we must discount other features, including chromosomes, and select genitalia as definitive. But consider a person who has an $\mathrm{XY}$ karyotype and is morphologically female (due to complete androgen insensitivity syndrome). It's not clear whether this person is male or female. Indeed, there doesn't seem to be a factual basis on which to arbitrate the question. But postoperative transsexuality seems exactly analogous. In both cases, there's no fact of the matter as to what sex or gender the person belongs to. One way to put the point is to say that a genitally postoperative transsexual woman is actually (ironically) positioned problematically with respect to the binary. To be sure, one can argue that all things being equal, one should defer to self-identity claims. But at best, the genitally post-op trans person is admitted into the category woman only marginally, owing to a decision to take her self-identity seriously. Besides that, she's actually very close to the border between male and female.

The stronger version fares even worse. It's far from clear that a transsexual woman is really female or a woman prior to genital reconstruction surgery. This claim is made on the basis of the idea that an innate identity determines the question of which sex one actually belongs to. Yet is it not clear that this one feature should trump all the others in the case of sex assignment. And it's not clear what the argument is for saying that the body (genitalia, gonads, etc.) is defective while the internal sense of self is not. Why not say, as the psychological variant claims, that it's the gender identity instead that is problematic? Why not avoid saying that anything is wrong at all? The truth is, a transsexual woman prior to genital reconstruction surgery would most likely be viewed as really a man, at least in many mainstream contexts. ${ }^{8}$

\footnotetext{
${ }^{7}$ Serano (2007) defends this view (166). Her theory blends both models through her appeal to "subconscious sex" and the "intrinsic inclination" model of sexual and gender diversity $(99-100)$.

${ }^{8}$ There could be more give here than I'm allowing, especially in light of public recognition of intersex individuals (particularly in athletics).
} 
The point I'm pressing is that transsexual claims to belong to a sex do not appear to be metaphysically justified: they are claims that self-identities ought to be definitive in terms of the question of sex membership and gendered treatment. They are therefore political in nature. And the problem is that there's no underlying political vision sustaining the support for definitive self-identities. The beyond-the-binary model doesn't sustain the centrality of self-identity — or at least it doesn't do so in a way that views such a move as at all politically laudable, since the truly resistant position, in this model, is to make a challenge from a position beyond the binary. And the wrong-body model, as we have just seen, likewise fails. One of the main questions I therefore want to ask is this: How might we provide the political underpinnings of such a move? How might we highlight it as a resistant claim in the face of oppression?

Providing such an undergirding is important for a second reason, namely, the articulation of a basis for a transfeminism - that is, a politics that focuses on the intersections of sexist and transphobic oppression. ${ }^{9}$ The problem is that neither the beyond-the-binary model nor the wrong-body model provides a way to theorize trans women as subject to sexual oppression (as women) and transphobic oppression (as trans) in ways that are fully blended. Once transgender people are seen as oppressed through the wrongful imposition of a binary, it's unclear how a transsexual woman can ever be seen as being oppressed as a woman at all (since she's "beyond the binary"), let alone in ways that blend the oppression of women and the oppression of trans people.

A wrong-body account could, by contrast, potentially explain how a trans woman is oppressed both as a woman and as trans. However, it has theoretical commitments that are deeply problematic from a feminist perspective. For an appeal to an innate gender identity is hard to abide in a feminist account. Many feminists have endorsed the view that the term "woman" names a social group, status, or role. Simone de Beauvoir famously wrote, "One is not born, but rather becomes, a woman. No biological, psychological, economic fate determines the figure that the human female presents in society; it is civilization as a whole that produces this creature" $(1952,201)$. If Beauvoir is right, gender identity can't be innate.

Consider this analogy: How can one take seriously the view that one's self-conception as a bingo player is innate given that bingo and therefore bingo players are cultural creations? To be sure, one might (conceivably) have innate skills that make one a good bingo player (and presumably one would be good at playing similar games as well). But the conception of oneself as a bingo player can't be innate, given that bingo itself is a con-

${ }^{9}$ See Koyama (2003) and Serano (2007) for different versions of transfeminism. 
tingent cultural phenomenon. The same is true in the case of gender. And given that the social category "woman" has served to oppress human female persons, we quickly see why a view positing a native gender identity is going to be problematic insofar as it naturalizes sexist cultural phenomena.

To be sure, if one were to claim that the categories male and female weren't culturally constructed, the positing of an innate sense of self as male or female wouldn't present this difficulty. One could give the view some plausibility by deploying the notion of an internal body image (a kind of sexed proprioceptive awareness of one's own body). This is the tack Prosser takes. However, feminist scholars such as Anne Fausto-Sterling (2000) have convincingly argued for the social construction of sex as well. So, even an appeal to a native sense of self as male or female is in serious trouble. Indeed, the view that transsexuals aren't women and men but, rather, females and males in the wrong bodies seems too subtle by half. Certainly most transsexuals who identify as male or female also identify as men or women. This is particularly acute since sex and gender terms are often used interchangeably in ordinary discourse.

The theory I defend, then, aside from providing a political undergirding for trans people's claims to belong within a particular binary category, aims to facilitate a genuinely intermeshing account of trans oppression and the oppression of women. This will enable me to provide a critique of the wrong-body account. This is important because while the traditional wrongbody account makes a political gesture in helping to secure transsexual identities as belonging within a particular binary category, it does so in a way that feeds the very oppression it opposes. Showing this should help to clarify why the new model I've outlined is a better political basis for understanding the resistance inherent in the self-identities of trans people, particularly for those who claim to be men and women.

\section{Thinking trans}

Many of the trans people I know identify as men or women or as trans men or women. Many of us have not undergone genital reconstruction surgery, and many of us do not want to; however, some of us have, and some of us do. Some of us have surgically altered our bodies in different ways (and some have not), some of us take hormones (and some do not), and some of us have had silicone injections (and some have not). For the most part, we believe our genital configurations don't undermine facts about who we are. For example, some of us trans women are very clear that we're unequivocally and entirely women (even female) while firmly disavowing any interest in genital reconstruction surgery. By contrast, some of us do 
not identify as men or women at all, and some of us take "trans woman" to mean someone who is indeed beyond the binary. Our self-identifications are generally complex and hard to pin down. Indeed, the very meanings of gender terms are not stable. They're both variable and contested.

Consequently, when a trans woman says "I'm a woman" and her body is precisely the kind of body taken to invalidate a claim to womanhood (in mainstream culture), the claim is true in some trans subcultures because the meaning of the word "woman" is different; its very meaning is under contestation (Bettcher 2012b). Indeed, in some trans subcultures, trans women can be taken as paradigmatic women rather than as women who are only marginally so (i.e., on the border of male and female). This contestation is not merely verbal, since it tracks a contrast in underlying gender practices: there can be situations in which a trans woman lives a rich and vibrant life as woman, has friends as a woman, is loved as a woman, inhabits a social milieu in which she is a woman (in a trans subculture), and perhaps experiences sexual violence as a woman while simultaneously being viewed as a man who lives as a woman in dominant culture. In that culture, for example, if she were incarcerated, she would be housed in jail as a man, with other men; her entire life as a woman could be obliterated. I understand this in terms of María Lugones's concept of "multiple worlds of sense":

Worlds are all lived and they organize the social as heterogeneous, multiple. I think of the social as intersubjectively constructed in a variety of tense ways, forces at odds, impinging differently in the construction of any world. Any world is tense, not just in tense inner turmoil but also in tense acknowledged or unacknowledged contestation with other worlds. I think that there are many worlds, not autonomous, but intertwined semantically and materially, with a logic that is sufficiently self-coherent and sufficiently in contradiction with others to constitute an alternative construction of the social. Whether or not a particular world ceases to be is a matter of political contestation. No world is either atomic or autonomous. Many worlds stand in relations of power to other worlds, which include a second order of meaning. (Lugones 2003, 21)

We can understand a trans world in resistant relation to a dominant world, where part of what is under contestation are alternative gender practices (including the semantics of gender terms). ${ }^{10}$ More precisely, we should rec-

${ }^{10}$ See Hale (1997) on this point. 
ognize a multiplicity of trans worlds in relation to a multiplicity of dominant ones.

Once we adopt a Lugonian framework for understanding trans oppression and resistance, we can see a serious problem inherent in both the wrong-body and transgender approaches: they take the dominant meanings of gender terms for granted, thereby foreclosing the possibility of multiply resistant meanings (Bettcher 2012b). In a beyond-the-binary model, to say that trans people are marginal with respect to the binary is to locate them in terms of the categories "man" and "woman" as dominantly understood. If trans bodies can have different resistant meanings, the decision to say of those bodies that they are "mixed" or "in between" is precisely to assume a dominant interpretation. So the problem is not the rigidity of the binary categories but rather the starting assumption that there is only one interpretation in the first place (the dominant one). Similarly, in the wrong-body model, to become a woman or a man requires genital reconstruction surgery as the correction of wrongness. But this is to accept a dominant understanding of what a man or a woman is.

Notably, if we frame trans in a Lugonian model, we're also able to allow for a deep intermeshing of multiple forms of oppression and resistance. For example, there's no guarantee that English gender terms need to have only English meanings. The word "transgender" as used and circulated within a world inhabited by Spanish-speaking trans women can have a secondorder (Spanish) meaning that recognizes and resists the English linguistic dominance backing the term. Such considerations concern the attempt to speak of various forms or axes of oppression that can sometimes intersect: insofar as the very expressions designating distinct modalities of oppression and resistance ("woman," "trans," "people of color") are subject to meaning contestations across the other alleged axes of oppression, the very attempt to fix a meaning may be a gesture of power. To separate the oppression of women from the oppression of trans people forecloses the way "woman" is subject to political contestation across a dimension of trans oppression and resistance.

That said, it's possible to speak of different forms of oppression, and it's possible to speak of different kinds of trans oppression in particular. Rather than focusing on abstract vectors of oppression defined only in terms of broad identity categories such "woman" or "trans," I focus on a very specific institutionalized form of transphobic violence. While it is not the only form of transphobic violence, it is an important one. And it is one that cannot be reduced to sexist or racist forms of violence, although it is essentially interwoven with them.

Recent literature has paid increasing attention to a specific form of transphobia whereby trans people are viewed as "deceivers"- people who try to 
pretend they are something they are not. I have elsewhere distinguished two representations of trans people (Bettcher 2006a): as deceivers (those who are discovered to be "really a man or woman," "disguised as a woman or man") and as pretenders (those who are already recognized as trans and seen as engaging in nothing but a kind of pretense). Similarly, Julia Serano $(2007,36)$ contrasts two media representations of trans women- "the sexualized deceiver" and "the pathetic transsexual" (the ridiculous man in a dress).

While my account is general, applying to trans men and trans women alike, Serano's focuses on trans women specifically. Together, the two accounts point to a general phenomenon that is manifested in a pronounced way for trans women. This gender asymmetry is underscored by the findings of Kristen Schilt and Laurel Westbrook (2009). Westbrook finds, in her analysis of media reports on 232 homicides of people "described as doing gender so as to possibly be seen as a gender other than the one they were assigned at birth," that in 56 percent of cases, the reporters "depict violence as resulting from private, sexual interactions in which the perpetrator feels 'tricked' into homosexuality by 'gender deceivers'" (Schilt and Westbrook 2009,452 ) and that 95 percent of all cases involved the killing of a trans woman by a nontrans man. Yet Schilt also finds, in her study of trans men in the workplace, that while women can in most cases accept trans men as men, "in sexualized situations, women frame transmen as deceptivetricking women into seemingly heterosexual relationships without the necessary biological marker of manhood" (Schilt and Westbrook 2009, 450). While the deceiver representation applies to trans men and trans women alike, it applies to trans women in more extreme ways.

Since the vast majority of trans women killed are trans women of color, trans women who are poor, and trans women who are sex workers, it is not enough to focus on transphobic violence across gender differences: race and class must likewise be centered (Namaste 2005; Bettcher 2006a; MacKenzie and Marcel 2009). For example, Gordene MacKenzie and Mary Marcel (2009) highlight the racist quality of media coverage of the US murders of Deborah Forte, Chanelle Pickett, Rita Hester, and Gwen Araujo: "Typically [women or color] are depicted as more exotic, wild, desirable, and out of control than their white counterparts" (84).

The role of sex work must also be seen as occupying a special role in our understanding of this kind of transphobia. Bella Evangelista and Chanel Larkin, for example, were murdered when a client or potential client learned of their transgender status (O'Bryan 2005; Erbentraut 2010). And, drawing on focus group data, Tooru Nemoto and his coauthors cite "general safety issues [for trans sex workers] related to being 'discovered' as a transgender" (Nemoto et al. 2004, 729). As one study respondent observed: 
"No one's going to kill a gay man if he finds a dick between his legs. No one's gonna kill a gay woman if he finds a pussy. But they will definitely put a knife through a tranny's throat if they see breasts and dick" (729).

Beyond this, Vek Lewis has shown how the deceiver representation can operate in a Latin American context: "A similar rhetoric operates in the inscription of travestismo or cross-dressing as elaborate charade or ruse. While such rhetoric often operates in both U.S. and Latin American contexts, the associations of criminality, however, are particularly strong in Latin America historically" $(2008,12-13)$. Lewis thereby highlights the need to understand the specific cultural situatedness of this kind of transphobia.

My own position is unique, however, in using the deceiver representation to rethink the very notion of doing gender (within the dominant culture). In my view, gender presentation represents genital status (Bettcher $2007,53)$. Or, as I would put it now, clothed gender presentation represents naked gender presentation through euphemistic means (Bettcher 2012a, 329-30). This is ironic since one of the main functions of clothing is to conceal the body. Yet it is precisely through this concealment that genital status is disclosed.

In cases when public gender presentation and private genitalia are construed as misaligned, this representational relation yields the conditions for what I will call reality enforcement-the basic type of transphobia grounding the deceiver representation. While there are many features associated with reality enforcement, it has four essential ones: identity invalidation, the appearance-reality contrast, the deceiver-pretender double bind, and genital verification. Identity invalidation is the erasure of a trans person's gender identity through an opposing categorization (e.g., a trans person sees herself as a woman, but she is categorized as a man). This invalidation is framed in terms of the appearance-reality contrast (e.g., a trans woman may be represented as "really a man disguised as a woman"). And this contrast is manifested in one of two ways that constitute a double-bind for trans people-namely, passing as nontrans (and hence running the risk of exposure as a deceiver) or else being openly trans (and consequently being relegated to a mere pretender). Genital verification can be a literal exposure (as with Brandon Teena, Gwen Araujo, and Angie Zapata) or else a discursive reveal through euphemistic comments like "was discovered to be anatomically male." These disclosures anchor identity invalidation in the notion of genitalia as a kind of concealed reality.

While reality enforcement might seem to affect only trans people who have not had genital reconstruction surgery, it can also affect those who have as well. According to Harold Garfinkel (1967), the common-sense folk view about sex (i.e., the "natural" attitude) is moral in nature (122). Genitalia are viewed as legitimate possessions, making it possible to speak of 
absent genitalia to which one is entitled (127). For example, a man who loses his genitals in an accident may speak of the penis that he was meant to have. Similarly, the surgically constructed genitalia of trans people can be transphobically viewed as at odds with the genitalia that nature intended. To capture this idea, I use the expressions "moral genitalia" and "moral sex." My thesis, then, is that public gender presentation euphemistically communicates moral genitalia. Typically, moral genitalia are actual genitalia, but they can also be the absent genitalia that nature intended. In this way, genitally postoperative trans people can also be subject to reality enforcement.

My thesis departs in important ways from some other theories of gender within the ethnomethodological tradition. Suzanne Kessler and Wendy McKenna (1978) attempt to build on Garfinkel's theories by introducing the notion of "cultural genitalia" (154). This concept is broader than Garfinkel's in including not only moral genitalia but also the genitalia one is merely presumed to have. This expansion is bound to their thesis that people become men and women through the process of having gender categories attributed to them (13) and that gender attribution is essentially genital attribution (154). What enables the conjunction of presumed and moral genitalia is their claim that gender attributions are almost impossible to overturn (17). Unfortunately, this claim is false, as the phenomenon of reality enforcement plainly shows. If their claim were true, reality enforcement would not occur with such frequency. Nor would it be a pervasive threat in the daily lives of trans people.

So we need a distinction between actual moral genitalia (often concealed) and the merely presumed moral genitalia that are attributed in cases of concealment. This has consequences for Candace West and Don $\mathrm{H}$. Zimmerman's (1987) classic account, which draws on Kessler and McKenna's work (132). West and Zimmerman distinguish between sex, assigned on the basis of biological criteria (including genitalia), and sex category, assigned on the basis of "doing gender" (127). The latter involves a kind of gender display - behaving in such a way so as to be categorized as a woman or a man, that is, to be held accountable to a certain set of norms (135). While they maintain that sex category stands as a kind of proxy for sex in everyday life, their notion of sex is a generically biological one (127). The notion of sex category, like the earlier notion of cultural genitalia, by contrast, is supposed to do the work of Garfinkel's notion of moral sex, effectively wedding presumed sex and moral sex together (West and Zimmerman 1987, 131-32).

What enables this move is the assumption that actual genital sex has virtually nothing to do with the negotiations of gender in everyday life (West and Zimmerman 1987, 132). But for trans people, the risk of real- 
ity enforcement (including genital verification) is most certainly part of the fabric of everyday life. So the everyday life that West and Zimmerman theorize is not that of trans people. Once we embed reality enforcement within the context of everyday gender practices, we recognize that moral sex ought to be wedded not to public appearance but to the concealed private body instead. That is, sex itself is moral in nature, while sex category is nothing more than the euphemistically displayed or merely apparent moral sex. Doing gender, then, is not merely the management of one's accountability to a set of gendered norms; it is a euphemistic display of one's concealed moral (genital) sex.

Crucially, both of these theories fail to explain moral genitalia, which are often concealed, because they take concealment for granted. While the convention of concealing genitals is acknowledged in both texts, it is in no way theorized. And everyday contexts in which genitals are indeed exposed (genital verification, sexual relations, public changing rooms, group housing, strip searches) are generally elided. Consequently what they fail to theorize is the moral character of concealment and exposure. For genital verification - a clear privacy violation - is surely a form of sexual abuse. This needs to be acknowledged and then theorized.

In my view, what explains moral genitalia (and the natural attitude in general) is the subjection of bodies to sex-differential boundaries of privacy and decency - boundaries essential to the very possibility of intimacy (Bettcher 2012a). For example, female nipples are subjected to boundaries on sensory access while male nipples are not. And while a man seeing a woman's genitals may constitute a violation of her privacy, a woman seeing a man's may involve his committing an indecency offense. These differential boundary structures yield two sex-differentiated forms of intimate self-presentation, that is, two sex-differentiated forms of nakedness (male and female) that contrast with the two sex-differentiated forms of public gender presentation (326).

The point is that my account illuminates reality enforcement (including its sexually abusive nature) as an aspect of everyday life for trans people, where earlier ethnomethdological accounts fail. And once the basis for reality enforcement is situated in gender, understood as an omnipresent, situated, and interactional accomplishment, it becomes easier to see, as Candace West and Sarah Fenstermaker (1995) propose, how race and class and other oppressions (as situated and interactional accomplishments) inhere in the same practices, thereby yielding experiences of gender, race, and class as simultaneous and wholly integrated. For example, I have argued elsewhere that the function of public gender presentation in communicating private genitalia is part of a larger system of nonverbal, nonconsensual com- 
munication that facilitates (hetero)sexual manipulation. Female gender behavior presentation sometimes has the function of communicating sexual interest (regardless of the actual intentions of the women herself), and it is through this that genital sex is thereby communicated. This can yield a multiple-bind scenario for trans women: "On the one hand, opting for invisibility and passing as non-trans may require that an MTF take up gender presentation that is generally 'yes'-encoded with respect to day-to-day negotiations of heterosexual sexuality. Failing to do so may leave MTFs open to 'exposure as a deceiver' and transphobic violence. Yet successfully taking up this presentation may also increase sexual scrutiny and the possibility of actual sexual interaction and therefore once again open MTFs to 'exposure as a deceiver'" (Bettcher 2006b, 207). In such a scenario, a trans woman will experience both standard sexual manipulation and the potential for transphobia as simultaneous and wholly integrated.

Acknowledging this, however, doesn't preclude pointing to the essential features of reality enforcement that recur across differences. While it's true that trans women of color are the group most often subject to extreme acts of violence as supposed sexual deceivers, this should not lead us to conclude that white trans men are somehow free from extreme acts of transphobia (think, e.g., of Brandon Teena). In fact, many are subject to less extreme variants of it, as Schilt's work shows (Schilt and Westbrook 2009). While these differences need to be made central, the need to focus on structural similitude remains imperative. To ignore similitude would mean losing sight of the larger picture that enables us to understand the workings of such violence. What is needed, rather, is a deeper account of how this form of transphobia is differentially distributed across multiple differences.

Since the most extreme cases of reality enforcement tend to occur where there is a maximal intermeshing of oppressions, I believe these cases ought to be understood as paradigmatic. Notably, we find other commonly associated features (sexualized context of discovery, sexual violence, murder, blaming the victim) in these cases. So an account of reality enforcement ought to begin with the premise that it is essentially an instantiation of racist violence, sexist violence, transphobic violence, and violence against sex workers all at once (roughly along the lines of my account of the fusion of reality enforcement and sexual manipulation in the case of trans women, discussed above). By identifying shared structural features across differences, one can outline a trajectory of transphobic violence: as variations of reality enforcement move away from paradigmatic cases, they will tend to lose some of the commonly associated features and hence become less severe or less likely. Since reality enforcement can be understood as a form 
of violence that cuts across racial, class, cultural, and gender differences differently, we can talk of a form of violence that cannot be reduced to sexism, racism, or classism while at the same time being fundamentally interwoven with all three.

While I do not believe that reality enforcement is the only way that trans people are oppressed, it's worth taking seriously for several reasons. First, given that the conditions for reality enforcement are immanent in the very accomplishment of gender, passing (as nontrans) is intimately connected to reality enforcement. To pass as a woman, in this view, is to successfully communicate that one is genitally female. It's little wonder, then, that passing can be such a pressing and pervasive issue in the lives of many trans people. The question of whether to pass or not is significantly informed by a deceiver-pretender bind. In other words, the very pressure to pass is generated, in part, by not wanting to be viewed as a mere pretender. And, of course, passing itself is fraught with worries about potential discovery (or "getting clocked"). These worries are particularly pressing in the context of dating (when the potential date doesn't know that one is trans).

Second, reality enforcement is a precondition of the transphobia that arises when someone is taken to engage in gender-nonnormative behavior. If a person is subjected to discrimination or violence because this person is taken to be in violation of gender norms (perhaps because the person is wearing a dress), this is no doubt because the person is being transphobically viewed as a man. But if this person sees herself as a woman, then she won't see herself as violating a gender norm. That is, whether a person can be held in violation of a norm requires, as a precondition, that that person be interpreted as subject to that norm in the first place. And people are generally taken to be subject to gender norms based on their moral sex. Reality enforcement, therefore, is a precondition of being viewed as a rule violator. Of course, there's very good evidence that discrimination against trans people leads to all sorts of devastating material consequences. For example, the recent study "Injustice at Every Turn" found that 90 percent of the respondents (all of whom were trans) reported discrimination in the workplace, that respondents were almost four times more likely than nontrans people to live in a households with an income of less than $\$ 10,000$ a year, and that 71 percent of respondents "attempted to avoid discrimination by hiding their gender or gender transition" (Grant, Mottet, and Tanis 2011). The last finding is noteworthy because it underscores the role of passing in the lives of trans people and shows that discrimination is predicated on being interpreted in a particular way.

Third, reality enforcement is institutionalized. This can be seen by considering various forms of sex segregation-including of public restrooms and changing rooms, domestic violence shelters, homeless shelters, shared 
hospital rooms, and jails and prisons, as well as policies enforcing same-sex searches by police officers and other security officials. In all cases, we find the blending of reality enforcement with sexual violation. Consider that the underlying moral motivation for such segregation is often to protect women from both violations of privacy and offenses of indecency (committed by men). Ironically, then, when trans women are housed according to perceived genital status (by appeal to such moral motivation), they are subject to the very same violations of privacy and offenses of indecency. For trans women, sex segregation yields boundary violations as a constitutive part of identity enforcement.

Finally, the attempt to push back against the pervasive social threat of reality enforcement can lead trans people to put themselves in harm's way. Some trans women may stay in abusive relationships to prove their womanhood, and some trans men may engage in abusive behavior to prove their masculinity. Studies have shown, for example, that some trans women may engage in unprotected sex (both with customers and primary partners) to secure validation as women, thereby increasing risk for HIV infection (Nemoto et al. 2004). Moreover, it isn't uncommon for there to be pecking orders among groups of trans women on the basis of who is more "real." Such an order can be established on the basis of surgical modification, achievement of certain beauty standards, or ability to pass. Unfortunately, such behavior can lead us to treat each other badly - even with violence.

That said, while reality enforcement is clearly a tremendously important aspect of trans life, it doesn't fit within the beyond-the-binary model. Not only do many victims of reality enforcement identify as men or women, but the perpetrators see their victims as women or men and the appearancereality contrast is likewise situated within the binary ("really a man dressed as a woman"). So this kind of transphobia doesn't concern anything beyond the binary; it concerns where in the binary somebody is located. (Contrast this with cases in which trans people are dismissed as "its"; such cases do seem to concern people deemed to be outside of the binary.) Rather than a consequence of overly rigid gender boundaries that eliminate those who are categorically liminal, this form of violence is linked with notions of appearance, illusion, discovery, reality. As we'll now see, it is precisely this framework that can allow us to see the wrong-body narrative as resisting trans oppression.

\section{Resistance in the wrong-body narrative}

Consider the view that gender identity is innate, in opposition to the theoretical claim that sex and gender are socially constructed. We can see why constructionism might be particularly grating in light of reality enforce- 
ment: if trans people are systematically subject to allegations of deception or pretense, the idea that sex and gender are constructs might seem especially threatening. To be sure, one could reply that everybody's sex and gender is a construction-the claim isn't specific to trans folk. But this would miss the unique way trans people are constructed as constructions.

Consider: If all the world's a stage on which we all play a part, trans individuals play actors. For somebody frustrated at being constructed as an actor, the mere claim that everybody is actually an actor would, by itself, erase the distinctive and oppressive way in which one was specifically constructed as an actor; it would provide no help in undermining being specifically constructed as an actor; and it would reinforce the claim that one was indeed an actor while obscuring the fact that such a reinforcement was being made.

One might reply that to call something constructed is not to call it unreal. Bingo, while socially constructed, exists. However, constructionism exposes sex and gender as artificial in light of their originally being taken as natural. This gives us not only a distinction between the socially constructed and the biologically given; it shows us a social construction that passes itself off as something natural, a fraudulent social construction. And it's this representation that is particularly concerning in light of reality enforcement. The concern is only aggravated when transgender phenomena are utilized as argumentative tools to show that sex and gender are constructed in this deceptive way or, worse, when trans people are reduced to tropes of this misleading construction (see Namaste 2000). It's hard to believe such uses aren't ultimately trading on representations of trans people as deceivers or pretenders.

This might seem to place transsexual politics and feminist politics at odds with each other, with one group wanting to naturalize, the other wanting to denaturalize. And that's terribly unfortunate since efforts at naturalizing go against the interests of trans women as women and are therefore problematic from a transfeminist perspective. The mistake, however, is to focus on only one modality of oppression as abstracted from others. The wrong-body account falls prey to this difficulty. In order to resist a reduction to mere pretense, some trans people affirm a reality that's based on an appeal to an innate gender identity. While the affirmation is responsive to trans oppression, it undercuts trans women by naturalizing sexist gender differences.

Part of the answer is to support a constructionism that recognizes the nonreducibility of trans oppression and resistance. In particular, such a theory should recognize that trans people are constructed as frauds and that some trans people respond by appealing to gender realness. This re- 
quires distinguishing between actual acts of resistance and a theory that illuminates that resistance. While actual acts of resistance may appeal to gender realness (and use a theory that justifies that realness), a constructionist theory can be used to illuminate such maneuvers without itself committing to the realness of gender. Moreover, while the actual appeal to native gender must be rejected from a transfeminist perspective, the socially constituted denial of realness must be taken with dead seriousness. Indeed, this theory must identify and illuminate tactics of resistance to the denial of reality that avoid abstracting from the multiplicity of oppressions.

Beyond this, the strong version of the wrong-body narrative can actually be seen to dramatically reverse the denial of realness. While reality enforcement says "that's really a woman, merely dressed as a man," the wrongbody narrative says "that's really a man, disguised by a misleading body." In both cases there's an appearance-reality contrast - either between gender presentation and sexed body or between sexed body and gender identity. Once placed in this context, the identity claim "I'm really a woman" (determined by identity) can be seen to directly contest the claim "You're really a man" (determined by genitalia). There's a shift from hidden genitalia to hidden identity. Through this shift, accusations of deception and pretense are undermined. Behind the gender presentation is a sexed body. But behind the sexed body is a sexed identity. So while one might say that gender presentation misrepresents the sexed body, it turns out that the sexed body actually misrepresents a more deeply concealed identity: an identity behind a body behind a presentation. The result is that the two misrepresentations cancel each other out, and the gender presentation turns out to correctly represent the sexed body one was meant to have.

This requires a rethinking of the view that trans people who endorse this narrative are doing nothing more than endorsing the natural attitude about sex (Garfinkel 1967). While it is true that such trans people are effectively claiming a moral sex in order to see their actual genitals as illegitimate and to speak of the genitalia to which they are entitled, they are also reversing the denial of realness that flows from the representational relation between public gender presentation and intimate presentation-the very relation founding the natural attitude. Through this reversal, the socalled truth about gender (one's naked body) becomes nothing more than a misleading appearance, while concealed identity is now the locus of moral sex.

Thus, the body itself becomes deceptive. Rubin speaks, for example, of the common representations of bodies as betrayers $(2003,109)$. Once relegated to a mere appearance, the body becomes "wrong" insofar as it bears not only the normative weight of "not meant to be" but also the 
weight of misrepresenting moral sex. For Prosser, the material body ends up being viewed as a kind of second skin misrepresenting the "inner body" $(1998,82)$. When viewed this way, the concealed identity becomes a body itself - the real, hidden body (the body image). It's natural to think that a body image mirrors the literal body. However, in the wrong-body narrative, the literal body misrepresents the body image - that is, the body must be brought into alignment with the body image, not the other way around (Prosser 1998, 85). Here, we have a relation of misrepresentation between the encasing outside material body (that one has) and inside, ghostly, phenomenal body (that one was meant to have; Prosser 1998, 85).

Notably, the misleading material body is sometimes viewed as a garment (Prosser 1998, 82). And surgery isn't always viewed as a mere change of clothes. Instead, it's viewed as a shedding of the garment. Prosser writes that "surgery strips the body bare to what it should have been" (83). The contrast is striking: in cases of reality enforcement, there's movement from "misleading" concealment to exposure (genital verification). In this account of surgical intervention, we also have movement from "misleading" concealment to exposure (gender confirmation). While the first is a forced violation, the latter is a voluntary stripping off of that which constricts the self-a moment of "freedom at last" rather than a sexual assault.

Notably, however, once the mistake has been rectified, the liminal bodythe misrepresenting garment or second skin-disappears entirely, leaving only the sexed body in alignment with gender presentation. This means the wrong-body narrative refrains from positing a more general representational relation between body and identity. To do so would require that all intimate bodies be viewed as mere presentations and all gender identities as the source of moral sex. On the contrary, the new sense of realness attributed to the body requires that it not be viewed as a mere presentation but, rather, the proper source of moral sex. Since the narrative leaves the general relation of representation between gender presentation and sexed body intact, it protects only those to whom it is taken to apply; it leaves other trans people still vulnerable to reality enforcement.

For example, an MTF transsexual may claim to have always been a woman (trapped in the wrong body) in contrast to an MTF cross-dresser who is still transphobically taken as really a man dressing as a woman, even though this may not reflect this person's own ways of self-identifying. Both cross-dresser and transsexual may be vulnerable to reality enforcement. But the wrong-body narrative requires a contrast between "the true transsexual" and "the mere cross-dresser" in order to work. It needs to leave the original appearance-reality contrast in place in order for the resistant appearance-reality contrast to reverse it: the cross-dresser as mere 
appearance, the transsexual trapped in the wrong body as the hidden reality. The former bears the weight of the deceiver-pretender representation in order to provide "the real transsexual" with this contrast: appearances to the contrary, this is not a man dressed as a woman but really a woman trapped in the body of man.

The force of the word "cross-dresser" cannot be underestimated. It is often reductively taken as a mere equivalent to "man who dresses as a woman." And it's used against trans people, regardless of how we self-identify. Consider, for example, Chanel Larkin, an African American trans woman who had turned to sex work to make ends meet and who was killed by a potential customer when she disclosed her trans status. The news media circulated representations of her as a "cross-dressing prostitute" and "a man posing as a woman" (Erbentraut 2010). The force of such expressions obviously inscribes an appearance-reality distinction that functions to stigmatize.

That the wrong-body narrative leaves this representational relation intact arises, in part, from the fact that it attempts to foreclose multiple (otherworldly) interpretations of terms like "woman." Once we recognize trans subcultures with alternate gender practices - practices in which gender presentation does not mean genitalia and in which bodies are encoded with intimacy boundaries in alternative ways - we can see how, in the wrong-body narrative, gender terms are largely interpreted via the mainstream practice of representing genital status through gender presentation. This is obvious in the weak version, which identifies womanhood with a particular sort of genitally based anatomy. But it's also true in the strong version of the narrative. For while this version does allow that innate gender identity alone can determine sex membership, such an identity is required to include a desire for genital reconstruction surgery to correct the wrong body, thereby bringing gender presentation and sexed body into proper alignment.

Yet it is precisely this foreclosure of the multiplicity of interpretations that allows for abstraction from the multiplicity of oppressions. Consider, for example, that some of the technologies of bodily reconstruction cost a lot of money and, when not covered by a health care system, are out of reach for most trans people. Note, also, that in those trans women's subcultures where sex work is a dominant presence, genital reconstruction surgery will not necessarily be a desired goal, since this may well cause the loss of a crucial source of income. More generally, desires are shaped by what is envisioned as possible and also by what is valued within a given local subculture; some trans subcultures simply don't center genital reconstruction surgery at all. Other body modifications (such as breast augmenta- 
tion or facial reconstruction) are more central. For example, the use of silicon pumping (the injection of silicon directly into the body) may be a far more significant issue in some community contexts. ${ }^{11}$

Since the wrong-body narrative is deeply connected to genital reconstruction technologies, the narrative, just as the technologies, is open to worries about class- and race-differentiated access. The wrong-body narrative outlines a standard telos (genital reconstruction surgery), and any identity that fails to desire that telos is ruled ineligible. It thereby attempts to restrict access to womanhood (or manhood) itself through hegemonic class-, race-, and culture-inflected modalities.

Ironically, the wrong-body model can never quite free itself from the source of oppression that it has sought to contest through reversal. This is because its resistance appears to occur in the very same worlds that are trans oppressive. One consequence has been the historically rigid clinical criteria instituted to determine whether a trans person is "a true transsexual" (see Prosser 1998, 110-12). Insofar as the problem is framed as an identity-body misalignment, a method for determining whether a trans person really does have the identity she claims to have seems to be required. For if she does not, the danger is that she will turn out to be a gender deceiver-that is, merely a cross-dresser.

Unsurprisingly, clinicians have historically viewed trans people with suspicion, fearing the possibility of "fraudulence" (Prosser 1998, 111). Here the fear doesn't concern misrepresentation of genital status through gender presentation. Rather, it concerns outright lying in the therapeutic context through falsification of narrative. The recurrence of this theme is hardly an accident. A trans person who is considered not to be a genuine transsexual is left without recourse to the helpful intervention of the wrongbody discourse and therefore relegated to the status of gender deceiver. That is, a fraudulent transsexual is nothing but a mere cross-dresser-our original gender deceiver who is trying to pass as a genuine transsexual in order to pass as a woman or man. It is this possibility that helps facilitate an obsessive concern with detecting authenticity, facilitated by the presumption that trans people are potential gender deceivers until proven otherwise.

In the clinical domain, trans autobiographical narratives necessarily function as a kind of identity representation; trans people are expected to tell the truth about their inaccessible gender identity in a way that mirrors gender presentation's supposed representation of genital status. This is unsurpris-

${ }^{11}$ Thanks to Alexis Rivera for this point. 
ing. Given that the body is taken to potentially misrepresent the hidden identity, the hidden identity now becomes the locus - that is, the locus of moral sex. Curiously, genital status and identity are alike in that they are subject to the boundaries of privacy and are therefore hidden away as concealed realities. So, when seen in light of the obsessive scrutiny and presumptive distrust they involve, these clinical authentication procedures start to look a lot like analogs of the genital verification procedure: false narratives are stripped away to reveal the naked truth about the deceptive cross-dresser.

This clinical distrust may generate circumstances in which trans people are perversely led to falsify their histories in order to access medical technologies. Prosser writes that it is in part this tendency among "some clinicians to approach the transsexual as a suspect text . . that may provoke transsexuals to 'falsify' histories in the first place" (1998, 111). Thus, insofar as the authentication procedures are rigidly determined in order to weed out potential gender deceivers, a double bind is created: either lie to the clinician or tell the truth and be relegated to the domain of gender fraudulence. This is simply a derivative variant of the original bind that confronts trans people: either pass as the opposite sex (i.e., be a gender deceiver) or come out as trans and be read as openly fraudulent (i.e., as a mere pretender). It's a lose-lose situation that occurs first on the street and then in the clinician's office.

While the wrong-body narrative may reverse and thereby contest reality enforcement, the fact that it leaves the representational relation between gender presentation and the sexed body intact means not only that anyone not deemed to be in the wrong body will be left out but also that the mechanics of reality enforcement will be replicated in the clinician's office. The abuse will hunt those "trapped in the wrong body" wherever they go.

What is needed, in my view, is fuller opposition to the very basis of reality enforcement - namely, the entire representational relation between gender presentation and genitalia. And this requires recognizing the existence of multiple worlds of sense, worlds in which terms such as "woman" have different, resistant meanings; worlds in which there exist different, resistant gender practices. This allows for the possibility that trans individuals who self-identify as men or women are every bit as resistant as those who do not. The deployments of identity categories in these contexts involve radical departures from dominant practices of gender and hence an alteration in the meaning of terms such as "woman" and "man." Now that we have uncovered reality enforcement as a kind of trans oppression, we can view this claiming of identity as analogous to the use of political ex- 
pressions such as "womyn" and "women of color"-expressions invested with meanings (or perhaps potentials for meaning) that are both lived in resistant opposition to dominant meanings and at the same time open ended and multiple. ${ }^{12}$ In doing so, we can see that by grounding trans oppression and resistance in a transgender model that necessarily locates the resistant potential of trans people beyond the binary, we've actually been trapped in the wrong theory.

Department of Philosophy

California State University, Los Angeles

\section{References}

Beauvoir, Simone de. 1952. The Second Sex. Ed. and trans. H. M. Parshley. New York: Random House.

Bettcher, Talia Mae. 2006a. "Appearance, Reality and Gender Deception: Reflections on Transphobic Violence and the Politics of Pretence." In Violence, Victims, Justifications: Philosophical Approaches, ed. Felix Ó Murchadha, 175-200. Oxford: Peter Lang.

- 2006b. "Understanding Transphobia: Authenticity and Sexual Abuse." In Trans/forming Feminisms: Transfeminist Voices Speak Out, ed. Krista ScottDixon, 203-10. Toronto: Sumach.

- 2007. "Evil Deceivers and Make-Believers: Transphobic Violence and the Politics of Illusion." Hypatia 22(3):43-65.

_. 2012a. "Full-Frontal Morality: The Naked Truth about Gender." Hypatia 27(2):319-37

- 2012b. 'Trans Women and the Meaning of 'Woman.'” In The Philosophy of Sex: Contemporary Readings, ed. Nicholas Power, Raja Halwani, and Alan Soble, 233-50. 6th ed. Lanham, MD: Rowman \& Littlefield.

Bornstein, Kate. 1994. Gender Outlaw: On Men, Women, and the Rest of Us. New York: Routledge.

Cooke, Suzan. 2007. “Goodbye to Transgender and All That.” TS-Si, May 7. http:// ts-si.org/guest-columns/2347-good-bye-to-transgender-and-all-that.

Erbentraut, Joseph. 2010. "Transgender Woman Murdered on Milwaukee Street." Edge, May 20. http://www.edgeboston.com/index.php?ch=newsš\&sc2=news \&sc3 $=$ \&id $=105882$.

Fausto-Sterling, Anne. 2000. Sexing the Body: Gender Politics and the Construction of Sexuality. New York: Basic.

Feinberg, Leslie. 1993. Stone Butch Blues: A Novel. Ithaca, NY: Firebrand.

${ }^{12}$ On "womyn," see Scheman $(1997,141)$; on "women of color," see Lugones (2003, $84)$. 
Garfinkel, Harold. 1967. Studies in Ethnomethodology. Englewood Cliffs, NJ: Prentice-Hall.

Grant, Jaime M., Lisa A. Mottet, and Justin Tanis. 2011. "Executive Summary: Injustice at Every Turn: A Report of the National Discrimination Survey." With Jack Harrison, Jody L. Herman, and Mara Keisling. Report, National Center for Transgender Equality and National Gay and Lesbian Task Force, Washington, DC. http://transequality.org/PDFs/NTDS_Exec_Summary.pdf.

Hale, C. Jacob. 1997. "Leatherdyke Boys and Their Daddies: How to Have Sex without Women or Men." Social Text 52/53 15(3/4):223-36.

Kessler, Suzanne J., and Wendy McKenna. 1978. Gender: An Ethnomethodological Approach. New York: Wiley.

Koyama, Emi. 2003. "The Transfeminist Manifesto.” In Catching a Wave: Reclaiming Feminism for the 21st Century, ed. Rory Dicker and Alison Piepmeier, 244-59. Boston: Northeastern University Press.

Lewis, Vek. 2008. "Of Lady-Killers and 'Men Dressed as Women': Soap Opera, Scapegoats and the Mexico City Police Department.” Portal 5(1):1-28. http:// epress.lib.uts.edu.au/journals/index.php/portal/article/view/480/594.

Lugones, María. 2003. Pilgrimages/Peregrinajes: Theorizing Coalition against Multiple Oppressions. Lanham, MD: Rowman \& Littlefield.

MacKenzie, Gordene, and Mary Marcel. 2009. "Media Coverage of the Murder of U.S. Transwomen of Color." In Local Violence, Global Media: Feminist Analyses of Gendered Representations, ed. Lisa M. Cuklanz and Sujata Moorti, 79-106. New York: Peter Lang.

Meyerowitz, Joanne. 2002. How Sex Changed: A History of Transsexuality in the United States. Cambridge, MA: Harvard University Press.

Namaste, Viviane K. 2000. “'Tragic Misreadings': Queer Theory's Erasure of Transgender Subjectivity." In Invisible Lives: The Erasure of Transsexual and Transgendered People, 9-23. Chicago: University of Chicago Press.

- 2005. Sex Change, Social Change: Reflections on Identity, Institutions, and Imperialism. Toronto: Women's Press.

Nemoto, Tooru, Don Operario, JoAnne Keatley, and David Villegas. 2004. "Social Context of HIV Risk Behaviours among Male-to-Female Transgenders of Colour." AIDS Care 16(6):724-35.

O'Bryan, Will. 2005. "Killer Sentenced in Transgender Murder Case." Metro Weekly, December 22. http://www.metroweekly.com/news/?ak=1905.

Prosser, Jay. 1998. Second Skins: The Body Narratives of Transsexuality. New York: Columbia University Press.

Rubin, Henry. 2003. Self-Made Men: Identity and Embodiment among Transsexual Men. Nashville, TN: Vanderbilt University Press.

Salamon, Gayle. 2010. Assuming a Body: Transgender and Rhetorics of Materiality. New York: Columbia University Press.

Scheman, Naomi. 1997. "Queering the Center by Centering the Queer: Reflections on Transsexuals and Secular Jews." In Feminists Rethink the Self, ed. Diana Tietjens Meyers, 124-62. Boulder, CO: Westview. 
Schilt, Kristen, and Laurel Westbrook. 2009. "Doing Gender, Doing Heteronormativity: 'Gender Normals,' Transgender People, and the Social Maintenance of Heterosexuality." Gender and Society 23(4):440-64.

Serano, Julia, 2007. Whipping Girl: A Transsexual Woman on Sexism and the Scapegoating of Femininity. Emeryville, CA: Seal.

Stone, Sandy. 1991. "The Empire Strikes Back: A Posttransexual Manifesto.” In Body Guards: The Cultural Politics of Gender Ambignity, ed. Julia Epstein and Kristina Straub, 280-304. New York: Routledge.

West, Candace, and Sarah Fenstermaker. 1995. "Doing Difference." Gender and Society $9(1): 8-37$.

West, Candace, and Don H. Zimmerman. 1987. "Doing Gender." Gender and Society $1(2): 125-51$. 